\title{
Beta-cell sensitivity to glucose is impaired after gastric bypass surgery
}

Marzieh Salehi, MD¹, Amalia Gastaldelli, $\mathrm{PhD}^{2}$, and David A. D’Alessio, MD

1 University of Cincinnati College of Medicine, Department of Medicine, Cincinnati, $\mathrm{OH}$; 2 Cardiometabolic Risk Unit, CNR Institute of Clinical Physiology, Pisa, Italy

Short title: $\beta$-cell glucose sensitivity after gastric bypass

Corresponding author and the reprint requests should be addressed to:

Marzieh Salehi, MD

marziehsalehi1@gmail.com

Key words: $\beta$-cell glucose sensitivity, gastric bypass surgery, glucose tolerance

Number of words, abstract: 196 and manuscript: 3395; Number of references: 43;

Number of figures: 3; Number of tables: 2

\section{Abstract}

Aims: Patients with Roux-en-Y gastric bypass surgery (GB) have exaggerated postprandial insulin secretion, which has been attributed to increased meal glucose appearance and enhanced incretin effect. Here we sought to determine $\beta$-cell glucose sensitivity in the absence of meal stimulation and insulinotropic gut factors.

Materials and methods: Twelve non-diabetic subjects with prior GB, and 7 matched non-surgical controls with normal glucose tolerance were studied. Blood glucose and been through the copyediting, typesetting, pagination and proofreading process, which may lead to differences between this version and the Version of Record. Please cite this article as doi: 10.1111/dom.13165 
insulin secretion rates were measured during a graded glucose infusion at increasing and then decreasing rates. Insulin sensitivity $\left(\mathrm{S}_{\mathrm{I}}\right)$ and glucose effectiveness $\left(\mathrm{S}_{\mathrm{G}}\right)$ were determined by the minimal model.

Results: The GB subjects had comparable $S_{1}$ to the controls. The GB subjects had relative hyperglycemia during highest dose of glucose infusion associated with significantly reduced $\beta$-cell glucose sensitivity throughout both step-up (GB: $34 \pm 6, C N$ : $82 \pm 9$ pmol.min $\left.{ }^{-1} \cdot \mathrm{mM}^{-1} . \mathrm{L}, \mathrm{p}<0.0001\right)$ and step-down (GB: $31 \pm 6, \mathrm{CN}: 74 \pm 9$ pmol.min $\left.{ }^{1} . \mathrm{mM}^{-1} . \mathrm{L}, \mathrm{p}<0.0001\right)$ phases of the glucose infusion. The GB subjects also had reduced $\mathrm{S}_{G}\left(\mathrm{~GB}: 0.04 \pm 0.00, \mathrm{CN}: 0.07 \pm 0.01 \mathrm{~min}^{-1}, \mathrm{p}=0.004\right)$.

Conclusion: In the absence of enteric stimuli $\beta$-cell sensitivity to changes in glycemia is blunted among individuals with GB, indicating a significant shift in a fundamental property of $\beta$-cell function several years after surgery.

\section{Introduction:}

The use of bariatric surgery has steadily increased in the U.S. in response to the rising prevalence of obesity. Added impetus for procedures such as gastric bypass (GB) comes from the now clear benefits on glycemic control in patients with type 2 diabetes [1-3]. Weight loss following GB leads to substantial improvement in insulin sensitivity [46], but there is also considerable evidence that surgical re-routing of nutrients through 
the gastrointestinal $(\mathrm{Gl})$ tract also improves post-meal insulin secretion in diabetic and non-diabetic individuals [7-10]. The mechanisms connecting surgical changes in the gut and $\beta$-cell function are not understood, but are a potential source of new targets for therapeutic development.

Assessment of prandial insulin secretion in GB subjects is complicated by increased rates of GI glucose appearance after surgery $[9,10]$. People with GB have substantially earlier and higher peaks of glucose after meal ingestion [11]. Along with wider prandial glycemic excursion, GB subjects have a massive release of the incretin glucagon likepeptide 1 (GLP-1) that is also shifted earlier in the postprandial course [11]. Indeed, GB increases the incretin effect in both diabetic and non-diabetic individuals[12, 13], and the effect of GLP-1 to stimulate insulin release increases more than 2-fold [13].

Modeling of plasma C-peptide concentrations in GB subjects after meals also suggests increased $\beta$-cell sensitivity to glucose $[7,14-16]$. However, the complexity of insulinotropic stimuli in surgical patients makes attribution of factors controlling insulin secretion difficult.

The aim of this study was to examine $\beta$-cell glucose sensitivity in GB subjects in the fasting state when the effect of meal stimulation or insulinotropic GI factors on insulin secretion is absent. We hypothesized that subjects with GB would have greater insulin responses to intravenous (IV) glucose-induced hyperglycemia than a matched control group.

\section{Materials and Methods}


Subjects: Twelve patients with previous history of GB and 7 obese control subjects with no previous history of $\mathrm{Gl}$ surgeries $(\mathrm{CN})$ were recruited in order of their presentation to clinic or response to advertisement. Both groups had a larger ratio of female to male participants similar to the background population of individuals with bariatric surgeries [17]. The GB individuals had fasting glucose levels less than $100 \mathrm{mg} / \mathrm{dl}$ and glycated hemoglobin $(\mathrm{HbA} 1 \mathrm{C})$ levels less than $5.7 \%$, and were enrolled at least 2 years after their operation when they had been weight stable for at least 6 months. Out of 12 patients after GB one had a history of well controlled type 2 diabetes treated with metformin without any related complication before surgery, and this resolved completely after surgery - 9 y prior to study; none of the GB subjects were taking anti-diabetic medications or drugs that affect glucose metabolism. The GB subjects achieved an average weight loss of $57 \pm 8 \mathrm{~kg}(19-100 \mathrm{~kg})$ over $6-12$ months after surgery and an average weight loss of $57 \pm 7 \mathrm{~kg}(8-82 \mathrm{~kg})$ over a mean of $6 \pm 1$ y (2-11 y) since surgery. The control subjects had no personal or family history of diabetes and had normal oral glucose tolerance tests before enrollment. Both $\mathrm{GB}$ and $\mathrm{CN}$ groups were matched for age, BMI, and fat mass. None of the subjects had renal dysfunction, or liver disorders. The institutional review board of the University of Cincinnati approved the protocol, and all participants provided written informed consent before the studies.

Experimental protocols: Subjects were instructed to follow their typical dietary habits and not engage in more than usual physical activity for 3 days before each visit. Participants were admitted to the General Clinical Research Center at Cincinnati Children's Hospital or the Clinical Research Unit at Cincinnati VA Medical Center in the morning after an overnight fast. Body composition was assessed using dual-energy X- 
ray absorptiometry and waist circumference was measured by a single staff member using a standardized procedure. Intravenous catheters were placed in each forearm for the withdrawal of blood and the infusion of $20 \%$ glucose; the arm used for blood sampling was continuously warmed to facilitate blood flow.

After removal of fasting blood samples, a graded glucose infusion was started at time 0 that followed scheduled increasing and decreasing rates $(0,4,8,16,8,4,0 \mathrm{mg} / \mathrm{kg} / \mathrm{min})$ at 40 minute intervals (Fig.1A). Thus, the first half of the experiment (40-160 min) achieved a progressive step-up in blood glucose, while the second (160-280 min) resulted in a gradual step-down. Blood samples were taken frequently every 5-10 min throughout the study (Fig. 1), immediately placed on ice, and plasma was separated within 60 minutes for storage at $-80^{\circ} \mathrm{C}$ until assay.

Assays: Blood samples were collected in tubes containing heparin for determinations of plasma insulin and blood glucose. Blood was also collected in tubes containing $50 \mathrm{mM}$ EDTA plus 500 kallikrein inhibitory units/ml aprotinin for measurement of plasma Cpeptide. Blood glucose concentrations were determined at the bedside using an automated glucose analyzer (YSI 2300 STAT Plus, Yellow Springs, OH). Insulin concentrations were determined using commercial ELISA (Alpco, Salem NH), and Cpeptide by commercial radioimmunoassay (Millipore, Billerica, MA), each according to the manufacturers' specifications.

Calculations and analysis: Fasting values of blood glucose and hormones were computed as the average of samples drawn from -15 to 40 min before initiation of the glucose infusion. Insulin secretion rates (ISR) were derived from plasma C-peptide concentrations using deconvolution with population estimates of C-peptide [18]; an 
assumption underlying this analysis is that gastric bypass does not affect C-peptide kinetics. Separate ISR:glucose slopes for the step-up and step-down portions of the protocol were generated for each individual using linear regression. Data were also fitted using the minimal model as previously described for the graded glucose protocol to measure insulin sensitivity and glucose effectiveness $[19,20]$.

Glucose, insulin, and ISR values during the graded glucose infusion were used to compute incremental areas under the curve (AUC) using the trapezoidal rule and are presented for times 40-160 min (step-up) and 40-280 min, the entire period of the study.

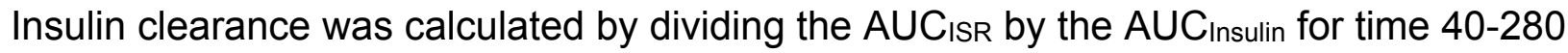
minutes $[21,22]$.

Statistical analysis: Data are presented as Mean \pm SEM. Baseline characteristics were compared using ANOVA or Chi-square tests. The parameters obtained from each subject in studies were compared among the two groups using ANOVA. Statistical analyses were performed using SPSS 23 (SPSS Inc., Chicago, IL).

\section{Results}

Characteristics of study subjects: The groups of GB and non-surgical subjects had comparable age, BMI, body composition, and waist circumference (Table 1). The controls had similar gender distribution and $\mathrm{HbA} 1 \mathrm{C}$ levels.

Glucose levels during graded glucose infusion: Fasting glucose levels were similar between the GB and CN groups, and the response to IV glucose administration overlapped until $120 \mathrm{~min}$ when infusion of the highest dose of glucose, $16 \mathrm{mg} / \mathrm{kg} / \mathrm{min}$, 
was started (Fig.1A). At this point the glucose curves for the two groups diverged and the glycemic peak was higher in the surgical subjects, although the AUC glucose for the step-up portion of the study did not differ (Table 2). During the descending phase of the glucose infusion, the GB subjects had higher nadir glucose concentrations (GB: $3.5 \pm$ $0.2, \mathrm{CN}: 2.8 \pm 0.1 \mathrm{mmol} / / ; \mathrm{p}=0.04$ ), and reached this point later than the controls. Over the course of the infusion protocol the overall glucose response (AUC Glucose $(40,280 \mathrm{~min})$ ) was significantly higher in the GB subjects (Fig.1A; Table 2).

$\beta$-cell responses to glucose infusion: In spite of matching for age, BMI, and body fat mass, the surgical subjects had significantly lower fasting insulin and ISR compared to the controls (Table 2). The integrated $\beta$-cell responses to increasing blood glucose, reflected as the AUC Insulin(40,160 min) and AUC ISR(40,160 min), were significantly lower in the GB group compared to the non-surgical controls (Fig.1B,C; Table 2). During the stepdown in the glucose ramp, insulin concentrations were also lower in the surgical group compared to the controls despite higher glucose levels (Fig.1C), while ISR in the two groups became more alike (Fig.1B). As a result AUC Insulin(40,280 min) was lower in the GB subjects, while the integrated insulin secretory response, AUC ISR(40,280 min), did not differ statistically from CN (Table 2). However, overall insulin secretion corrected for the ambient glucose concentration was significantly reduced after GB (AUC ISR(40,280 $\mathrm{min}) / \mathrm{AUC}$ Glucose $(40,280 \mathrm{~min})): 42 \pm 5$ in GB vs. $90 \pm 7$ in $\mathrm{CN}, \mathrm{p}=0.0001)$

$\beta$-cell sensitivity to glucose, measured as the average of the slopes of ISR:glucose for each subject, was significantly lower in the GB subjects during both ascending (GB: 34 $\pm 6, \mathrm{CN}: 82 \pm 9$ pmol.min ${ }^{-1} \cdot \mathrm{mM}^{-1} \cdot \mathrm{L}, \mathrm{p}<0.0001$ ) and descending phases (GB: $31 \pm 6$, $\mathrm{CN}: 74 \pm 9$ pmol.min ${ }^{-1} \cdot \mathrm{mM}^{-1} . \mathrm{L}, \mathrm{p}<0.0001$ ) of the glucose ramp (Fig.2A,B and Fig.3B). 
Subjects in both groups had higher insulin secretion rates per level of glucose during the step-down compared to step-up phase (Fig.3A). However, this difference, measured as the area inside the ascending and descending ISR:glucose response, was nearly twice as large in the GB subjects compared to the non-operated individuals (1211 vs. $868 \mathrm{pmol} / \mathrm{min}$ of ISR X mmol/l of glucose, Fig.3A).

Insulin sensitivity, glucose effectiveness, and insulin clearance: The insulin sensitivity index $\left(\mathrm{S}_{\mathrm{I}}\right)$ calculated from insulin and glucose concentrations using the minimal model was not significantly different between the two groups (GB: $4.6 \pm 1.2, \mathrm{CN}: 3.1 \pm 1.1\left[10^{-4}\right.$ $\left.\left.\mathrm{dL} \cdot \mathrm{kg}^{-1} \cdot \mathrm{min}^{-1} \cdot \mu \mathrm{U}^{-1} \cdot \mathrm{ml}\right]: \mathrm{p}=0.4\right)$. Glucose effectiveness, reflecting non-insulin mediated glucose disposition, was significantly less in the GB subjects than controls (GB: $0.04 \pm$ $0.00, \mathrm{CN}: 0.07 \pm 0.01 \mathrm{~min}^{-1}, \mathrm{p}=0.004$ ). The metabolic clearance rate of insulin (AUC ISR:Insulin(40,280 min)) during the study was slightly greater in surgical subjects compared to non-operated individuals (GB: $1.7 \pm 0.2, \mathrm{CN}: 1.0 \pm 0.2 ; p=0.06$ ) as previously reported by us [23] and others [24] using hyperinsulinemic clamp.

Among all the subjects there were no significant associations between $\beta$-cell glucose sensitivity, taken as the ISR:glucose slope up, and BMI, fasting glucose, or insulin sensitivity. Moreover, $\beta$-cell glucose sensitivity in the GB subjects was not correlated with amount of weight loss or time since surgery. This parameter was not also affected by prior history of diabetes in the single subject in the GB group whose $\beta$-cell sensitivity to glucose was the highest value among the GB subjects (Fig.3B).

\section{Discussion}


The hyperinsulinemia that accompanies rapid rates of prandial glucose appearance and clearance in GB patients has been taken as evidence that surgery enhances $\beta$-cell function. In fact, previous studies implicate increased incretin stimulation [12, 13] as well as greater $\beta$-cell glucose responsiveness after eating $[7,14-16]$ to explain higher rates of insulin secretion. The findings presented here support a dramatically different model of surgical effects on insulin secretion. In non-diabetic subjects studied several years after GB surgery, $\beta$-cell sensitivity to glucose in the absence of enteroinsular axis activity is substantially reduced compared to a well-matched non-surgical control group, suggesting that surgical effects on the $\beta$-cell response to glucose occur but not in the direction predicted by previous work. A plausible explanation for these findings is that dampening the insulin response to glucose is necessary to maintain homeostasis in the face of the rapid surges of nutrient and enteral $\beta$-cell stimuli that occur with each meal in GB patients.

We used a direct method of assessing $\beta$-cell sensitivity to glucose, applying a wellestablished and validated protocol [19, 25-29], infusing IV glucose in the fasting state, to eliminate the confounding from high rates of intestinal glucose appearance and incretin secretion that accompanies meal ingestion in GB. The up-and-down ramp provides a broad assessment of the insulin:glucose dose-response across a wide range of glycemia as well as a hysteresis pattern, first proposed by Breda et al [28] and later by Mari and Ferrannini [30], to describe insulin secretion with rising and falling blood glucose. While our study used a cross-sectional design rather than a prospective preand post-surgical comparison, our analysis is not confounded by the effects of massive changes in body weight and insulin sensitivity that occur in most subjects having GB. 
Insulin sensitivity measured using the method used previously during up- and downglucose infusion studies were similar in both surgical and non-surgical groups [19].

The major finding reported here, reduced $\beta$-cell glucose sensitivity and relative glucose intolerance in the GB subjects, was independent of insulin sensitivity, and evident acutely as glycemic stimulation increased. The difference in blood glucose between the GB and CN subjects was most evident with the highest rate of glucose infusion (16 $\mathrm{mg} / \mathrm{kg} / \mathrm{min}$ from 120-160 minutes), but insulin and ISR values diverged even earlier in the study, suggesting that the blunted $\beta$-cell response was the proximal factor, especially for the ascending phase. Besides the reduced ISR slope up in GB subjects, decreased $\beta$-cell glucose sensing is also supported by the flatter slope down of ISR as glucose levels wane, and the greater difference in ISR between the ramp-up and rampdown phases (hysteresis effect [28]). There has been a previous study of $\beta$-cell glucose sensitivity in non-diabetic GB subjects that also used a graded glucose infusion, but the authors did not observe differences compared to controls [29]. In that previous experiment a lower, more gradual glucose ramp was applied, which may have limited the capability to resolve differences such as those seen in our subjects. However, it is notable that the previously reported GB subjects tended to have a flatter ISR:glucose slope and slightly greater glycemic levels in response to IV glucose. Applying the upand-down protocol to GB subjects and a well-matched CN group, we noted slopes that were $50-60 \%$ less after surgery, and consistent enough within groups to be highly significant even with the modest number of subjects evaluated. Therefore, although this was a cross-sectional comparison, the results suggest that the GB procedure causes a significant change to a fundamental property of $\beta$-cells. 
Previously published work [7, 31, 32], including our own [11], using ingestion of meals or glucose to stimulate insulin secretion, contrasts with the results presented here. In general, results based on meal studies support stable or increased $\beta$-cell glucose sensitivity in non-diabetic subjects after GB along with improved insulin sensitivity [7, $31,32]$. However, these findings can be challenged because of the complexity of postprandial stimuli after surgery. We and other investigators have previously used IV glucose tolerance tests $[4,6-8,33-36]$ or hyperglycemic clamps $[13,37]$, to assess the impact of GB on $\beta$-cell response to glycemia. These studies have generally concluded that insulin response to IV glucose administration after surgery is stable, or improved when adjusted for changes in insulin sensitivity. While the relationship between glucosestimulated insulin secretion and insulin sensitivity has been long recognized, the association between the two parameters has only been described when the acute insulin response to glucose ingestion, or IV boluses of glucose are measured [38]. Notably, $\beta$-cell glucose sensitivity does not vary as a function of insulin sensitivity [30]. Moreover, since the graded glucose protocol captures insulin secretion in response to glucose alone, the attenuated response in the GB subjects is likely due to a $\beta$-cellautonomous adaptation to surgery.

Two obvious questions that arise when considering these results is how they fit with the unequivocal hyperinsulinemia of GB subjects in the first 60-90 minutes after eating, and why attenuation of $\beta$-cell glucose sensitivity would occur after surgery? While our data do not directly answer these questions we believe they are related, and can offer a speculation that at least gives some context to our observation. 
It is clear that after GB enteral glucose flux is changed dramatically, with estimates that gastric delivery to the intestine is increased up to 25 -fold [9], and appearance of meal glucose increased proportionately $[10,39,40]$. In addition, meal-stimulated GLP-1 is enhanced up to 20 -fold $[10,11]$. In the face of such extraordinary stimulation by circulating factors postprandial hyperinsulinemia is not surprising, even with a decrease in $\beta$-cell glucose sensitivity to $50 \%$ or less of normal as we show here. In fact, in a previous report in 10 non-diabetic patients after GB, normalizing the rate of nutrient delivery to the gut by administration of continuous infusion of 50 gram glucose solution directly into the Roux-limb over $50 \mathrm{~min}(4 \mathrm{kcal} / \mathrm{min})$ compared to 50 gram glucose ingestion over $3 \mathrm{~min}(\sim 70 \mathrm{kcal} / \mathrm{min})$ caused deterioration in glucose tolerance as a direct result of $50 \%$ reduction in $\beta$-cell sensitivity to glucose (insulin:glucose slope) [9]. Also we previously reported a study of a single subject with GB who had insulin secretion measured after meal ingestion, with passage through the gastric pouch into the jejunum, or after feeding by tube through her remnant stomach, with passage through the pylorus into the duodenum. In this subject meal appearance and $\beta$-cell glucose sensitivity was reduced by $30 \%$ with meal administration through gastrostomy compared to oral ingestion [41]. These observations are consistent with our current findings and interpretation in that once exaggerated enteral stimulation is removed $\beta$ cell function after GB is blunted.

A corollary to this argument is that if $\beta$-cell glucose sensitivity was not reduced, GB patients might have difficulty preventing significant drops in postabsorptive glycemia. Indeed, we have previously noted that GB subjects with the postprandial hypoglycemia syndrome had greater hysteresis effects [39], consistent with reduced $\beta$-cell sensitivity 
to declining glucose levels [28], compared to asymptomatic persons with GB or nonoperated controls. We hypothesize that following GB the $\beta$-cell adapts to mitigate excessive stimulation from the large surges of prandial secretagogues, and that people who do not make this adaptation develop hypoglycemia.

A second major finding from this study, and one that is also consistent with an adaptation to protect against hypoglycemia, is the reduction of glucose effectiveness after GB. A reduction of insulin-independent glucose disposal has been demonstrated previously in GB subjects studied with IV glucose tolerance tests [8]. In this report, diminished $\mathrm{S}_{\mathrm{G}}$ was observed only in individuals with normal glucose tolerance as opposed to those with preoperative impaired glucose tolerance or type 2 diabetes. Also reduction in $\mathrm{S}_{\mathrm{G}}$ occurred as early as one week after surgery and persisted for 3 months, suggesting that this change was not dependent on weight loss [8]. By contrast, a comparable amount of weight loss after dietary caloric restriction does not change glucose effectiveness [42]. It is also notable that individuals with post-GB hypoglycemia have higher $\mathrm{S}_{\mathrm{G}}$ during IV glucose tolerance tests than unaffected GB subjects [43]. Taken together these findings suggest the hypothesis that reduction in $\mathrm{S}_{\mathrm{G}}$ is part of general adaptation of glucose metabolism to prevent hypoglycemia after GB.

There are several limitations to our study that merit discussion. First, the sample size involved in this set of experiments is small and may not be reflective of the greater population of patients with GB. However, the GB group had the female predominance generally found in bariatric surgery cohorts, and there were not substantial effects of the time since surgery or weight loss on insulin secretion. Moreover, the effect size between the two groups was substantial with very little overlap in the parameters of insulin 
secretion. The cross-sectional design of the study is a second potential weakness as much of the groundbreaking work in the area of bariatric surgery is based on comparisons of subjects before and after surgery [1-5]. However, the benefit of withinsubjects designs is mitigated for interventions like GB that cause huge changes to metabolic physiology. Thus, for isolating a simple physiologic parameter, ie. $\beta$-cell sensitivity to glucose, there are advantages to well-matched cross-sectional cohorts. Finally, we did not perform meal tests on our cohort of patients without $\mathrm{GB}$, therefore, we cannot directly compare results from the graded glucose infusion to insulin secretion during enteral stimulation between the two groups. While this limits some of our interpretations, the disparity between previous work showing stable or enhanced insulin secretion in GB subjects after eating, with the current results demonstrating a marked reduction in $\beta$-cell glucose sensitivity is stark.

In summary, individuals with GB for 2-11 years given a fixed glucose infusion have reduced insulin responses and relative hyperglycemia. This blunted $\beta$-cell glucose sensitivity is independent of insulin sensitivity and was very consistent across our group of GB subjects. The change in a fundamental characteristic of $\beta$-cell function- the insulin response to glucose, in GB subjects with normal fasting glucose and $\mathrm{HbA1c}$ suggests an adaptation of $\beta$-cell function to surgery that accommodates glucose homeostasis to changes in $\mathrm{Gl}$ nutrient flux. The parallel reduction of $\mathrm{S}_{\mathrm{G}}$ could serve a similar function. These hypotheses require further study, but provide a novel perspective on glucose metabolism following gastric bypass.

\section{Acknowledgements}


We thank Leslie Baum and Brianne Reedy from Department of Medicine of University of Cincinnati for their technical support and nursing staff from Clinical Research Center of Cincinnati Children's Hospital and Clinical Research Unit of Cincinnati VA Medical Center for their expert technical assistance. We owe a great debt to our research participants. MS designed and supervised the study and, obtained the data, analyzed and interpreted the data, and wrote the manuscript; $A G$ analyzed the data; DAD and AG contributed to interpretation of data and review/editing of the manuscript. MS as the guarantor takes full responsibility for the work including the study design, access to data, and the decision to submit and publish the manuscript.

This work was supported by grants from the National Institute of Health, DK105379 (MS), DK083554 (MS), and DK101991 (DD) and in part by National Center for Advancing Translational Sciences, National Institute of Health grant 8 UL1 TR000077. Parts of this study were presented at the Endocrine Society's $96^{\text {th }}$ Annual Meeting, Chicago, IL. Authors have nothing to disclose conflicting with the content of current manuscript. 


\section{References}

[1] Mingrone G, Panunzi S, De Gaetano A, et al. Bariatric surgery versus conventional medical therapy for type 2 diabetes. N Engl J Med. 2012; 366: 1577-1585

[2] Ikramuddin S, Korner J, Lee WJ, et al. Roux-en-Y gastric bypass vs intensive medical management for the control of type 2 diabetes, hypertension, and hyperlipidemia: the Diabetes Surgery Study randomized clinical trial. Jama. 2013; 309: 2240-2249

[3] Schauer PR, Bhatt DL, Kirwan JP, et al. Bariatric Surgery versus Intensive Medical Therapy for Diabetes - 5-Year Outcomes. N Engl J Med. 2017; 376: 641-651

[4] Bojsen-Moller KN, Dirksen C, Jorgensen NB, et al. Early enhancements of hepatic and later of peripheral insulin sensitivity combined with increased postprandial insulin secretion contribute to improved glycemic control after Roux-en-Y gastric bypass. Diabetes. 2013:

[5] Bradley D, Conte C, Mittendorfer B, et al. Gastric bypass and banding equally improve insulin sensitivity and beta cell function. J Clin Invest. 2012; 122: 4667-4674

[6] Lin E, Liang Z, Frediani J, et al. Improvement in ss-cell function in patients with normal and hyperglycemia following Roux-en-Y gastric bypass surgery. Am J Physiol Endocrinol Metab. 2010; 299: E706-712

[7] Reed MA, Pories WJ, Chapman W, et al. Roux-en-Y gastric bypass corrects hyperinsulinemia implications for the remission of type 2 diabetes. J Clin Endocrinol Metab. 2011; 96: 2525-2531

[8] Martinussen C, Bojsen-Moller KN, Dirksen C, et al. Immediate enhancement of first-phase insulin secretion and unchanged glucose effectiveness in patients with type 2 diabetes after Roux-en-Y gastric bypass. Am J Physiol Endocrinol Metab. 2015; 308: E535-544

[9] Nguyen NQ, Debreceni TL, Bambrick JE, et al. Rapid gastric and intestinal transit is a major determinant of changes in blood glucose, intestinal hormones, glucose absorption, and postprandial symptoms after gastric bypass. Obesity (Silver Spring). 2014:

[10] Jacobsen SH, Bojsen-Moller KN, Dirksen C, et al. Effects of gastric bypass surgery on glucose absorption and metabolism during a mixed meal in glucose-tolerant individuals. Diabetologia. 2013; 56: 2250-2254

[11] Salehi M, Gastaldelli A, D'Alessio DA. Altered Islet Function and Insulin Clearance Cause Hyperinsulinemia in Gastric Bypass Patients With Symptoms of Postprandial Hypoglycemia. J Clin Endocrinol Metab. 2014: jc20132686

[12] Laferrere B, Heshka S, Wang K, et al. Incretin levels and effect are markedly enhanced 1 month after Roux-en-Y gastric bypass surgery in obese patients with type 2 diabetes. Diabetes Care. 2007; 30 : $1709-1716$

[13] Salehi M, Prigeon RL, D'Alessio DA. Gastric bypass surgery enhances glucagon-like peptide 1stimulated postprandial insulin secretion in humans. Diabetes. 2011; 60: 2308-2314

[14] Dutia R, Brakoniecki K, Bunker $\mathrm{P}$, et al. Limited recovery of beta-cell function after gastric bypass despite clinical diabetes remission. Diabetes. 2014; 63: 1214-1223

[15] Jorgensen NB, Dirksen C, Bojsen-Moller KN, et al. Exaggerated Glucagon-Like Peptide 1 Response Is Important for Improved beta-Cell Function and Glucose Tolerance After Roux-en-Y Gastric Bypass in Patients With Type 2 Diabetes. Diabetes. 2013; 62: 3044-3052

[16] Nguyen KT, Billington CJ, Vella A, et al. Preserved Insulin Secretory Capacity and Weight Loss Are the Predominant Predictors of Glycemic Control in Patients With Type 2 Diabetes Randomized to Rouxen-Y Gastric Bypass. Diabetes. 2015; 64: 3104-3110

[17] Santry HP, Gillen DL, Lauderdale DS. Trends in bariatric surgical procedures. Jama. 2005; 294: 1909-1917 
[18] Van Cauter E, Mestrez F, Sturis J, Polonsky KS. Estimation of insulin secretion rates from Cpeptide levels. Comparison of individual and standard kinetic parameters for C-peptide clearance. Diabetes. 1992; 41: 368-377

[19] Ehrmann DA, Breda E, Cavaghan MK, et al. Insulin secretory responses to rising and falling glucose concentrations are delayed in subjects with impaired glucose tolerance. Diabetologia. 2002; 45 : 509-517

[20] Bergman RN, Ider YZ, Bowden CR, Cobelli C. Quantitative estimation of insulin sensitivity. Am J Physiol. 1979; 236: E667-677

[21] Shuster LT, Go VL, Rizza RA, O'Brien PC, Service FJ. Incretin effect due to increased secretion and decreased clearance of insulin in normal humans. Diabetes. 1988; 37: 200-203

[22] Tillil H, Shapiro ET, Miller MA, et al. Dose-dependent effects of oral and intravenous glucose on insulin secretion and clearance in normal humans. Am J Physiol. 1988; 254: E349-357

[23] Salehi M, Woods SC, D'Alessio DA. Gastric bypass alters both glucose-dependent and glucoseindependent regulation of islet hormone secretion. Obesity (Silver Spring). 2015; 23: 2046-2052

[24] Bojsen-Moller KN, Dirksen C, Jorgensen NB, et al. Early enhancements of hepatic and later of peripheral insulin sensitivity combined with increased postprandial insulin secretion contribute to improved glycemic control after Roux-en-Y gastric bypass. Diabetes. 2014; 63: 1725-1737

[25] Ehrmann DA, Sturis J, Byrne MM, Karrison T, Rosenfield RL, Polonsky KS. Insulin secretory defects in polycystic ovary syndrome. Relationship to insulin sensitivity and family history of non-insulindependent diabetes mellitus. J Clin Invest. 1995; 96: 520-527

[26] Jones IR, Owens DR, Moody AJ, Luzio SD, Morris T, Hayes TM. The effects of glucose-dependent insulinotropic polypeptide infused at physiological concentrations in normal subjects and type 2 (noninsulin-dependent) diabetic patients on glucose tolerance and B-cell secretion. Diabetologia. 1987; 30: 707-712

[27] Toffolo G, Breda E, Cavaghan MK, Ehrmann DA, Polonsky KS, Cobelli C. Quantitative indexes of beta-cell function during graded up\&down glucose infusion from C-peptide minimal models. Am J Physiol Endocrinol Metab. 2001; 280: E2-10

[28] Breda E, Toffolo G, Polonsky KS, Cobelli C. Insulin release in impaired glucose tolerance: oral minimal model predicts normal sensitivity to glucose but defective response times. Diabetes. 2002; 51

Suppl 1: S227-233

[29] Kim SH, Abbasi F, Lamendola C, Reaven GM, McLaughlin T. Glucose-stimulated insulin secretion in gastric bypass patients with hypoglycemic syndrome: no evidence for inappropriate pancreatic betacell function. Obes Surg. 2010; 20: 1110-1116

[30] Mari A, Tura A, Natali A, et al. Impaired beta cell glucose sensitivity rather than inadequate compensation for insulin resistance is the dominant defect in glucose intolerance. Diabetologia. 2010; 53: 749-756

[31] Nannipieri M, Mari A, Anselmino $M$, et al. The role of beta-cell function and insulin sensitivity in the remission of type 2 diabetes after gastric bypass surgery. J Clin Endocrinol Metab. 2011; 96: E13721379

[32] Jorgensen NB, Jacobsen SH, Dirksen C, et al. Acute and long-term effects of Roux-en-Y gastric bypass on glucose metabolism in subjects with Type 2 diabetes and normal glucose tolerance. Am J Physiol Endocrinol Metab. 2012; 303: E122-131

[33] Morinigo R, Lacy AM, Casamitjana R, Delgado S, Gomis R, Vidal J. GLP-1 and changes in glucose tolerance following gastric bypass surgery in morbidly obese subjects. Obes Surg. 2006; 16: 1594-1601 [34] Polyzogopoulou EV, Kalfarentzos F, Vagenakis AG, Alexandrides TK. Restoration of euglycemia and normal acute insulin response to glucose in obese subjects with type 2 diabetes following bariatric surgery. Diabetes. 2003; 52: 1098-1103 
[35] Vidal J, Nicolau J, Romero F, et al. Long-term effects of Roux-en-Y gastric bypass surgery on plasma glucagon-like peptide- 1 and islet function in morbidly obese subjects. J Clin Endocrinol Metab. 2009; 94: 884-891

[36] Salinari S, Bertuzzi A, Guidone C, Previti E, Rubino F, Mingrone G. Insulin sensitivity and secretion changes after gastric bypass in normotolerant and diabetic obese subjects. Ann Surg. 2013; 257: 462-

468

[37] Dirksen C, Eiken A, Bojsen-Moller KN, et al. No Islet Cell Hyperfunction, but Altered Gut-Islet Regulation and Postprandial Hypoglycemia in Glucose-Tolerant Patients 3 Years After Gastric Bypass Surgery. Obes Surg. 2016; 26: 2263-2267

[38] DeFronzo RA, Tripathy D, Abdul-Ghani M, Musi N, Gastaldelli A. The disposition index does not reflect beta-cell function in IGT subjects treated with pioglitazone. J Clin Endocrinol Metab. 2014; 99: 3774-3781

[39] Salehi M, Gastaldelli A, D'Alessio DA. Blockade of glucagon-like peptide 1 receptor corrects postprandial hypoglycemia after gastric bypass. Gastroenterology. 2014; 146: 669-680 e662

[40] Camastra S, Muscelli E, Gastaldelli A, et al. Long-term effects of bariatric surgery on meal disposal and beta-cell function in diabetic and nondiabetic patients. Diabetes. 2013; 62: 3709-3717 [41] Salehi M, Gastaldelli A, D'Alessio DA. Evidence from a single individual that increased plasma GLP-1 and GLP-1-stimulated insulin secretion after gastric bypass are independent of foregut exclusion. Diabetologia. 2014; 57: 1495-1499

[42] Escalante-Pulido M, Escalante-Herrera A, Milke-Najar ME, Alpizar-Salazar M. Effects of weight loss on insulin secretion and in vivo insulin sensitivity in obese diabetic and non-diabetic subjects. Diabetes Nutr Metab. 2003; 16: 277-283

[43] Patti ME, Li P, Goldfine AB. Insulin response to oral stimuli and glucose effectiveness increased in neuroglycopenia following gastric bypass. Obesity (Silver Spring). 2015; 23: 798-807 


\section{Figure Legends}

Figure 1. (A) Blood glucose and glucose infusion rates, (B) insulin secretion rates, and (C) plasma insulin levels during up-and-down glucose infusion in subjects who underwent GB (solid line and closed circle, black bar) and nonsurgical controls (dashed line and open circle, white bar). The corresponding AUCs for 40 to 120 minutes are shown (insets). ${ }^{* *} p<0.001$ compared with GB. Data are presented as mean \pm SEM.

Figure 2. Slopes of ISR in response to blood glucose concentration during A) the stepup and B) step-down glucose infusion in GB (closed circle) and CN (open circles) subjects. Data are presented as mean \pm SEM.

Figure 3. A) $\beta$-cell sensitivity to glucose across the entire study in $\mathrm{GB}$ (closed circles) and $\mathrm{CN}$ (open circles) subjects, and B) individual slopes of the $12 \mathrm{~GB}$ and $7 \mathrm{CN}$ subjects during the increasing (up) and decreasing (dwn) glucose infusion. Solid arrow shows the ascending phase of glucose ramp, and hollow arrow the declining glucose phase. ${ }^{* *} p<0.001$ compared with GB. Data are presented as mean \pm SEM. 
Table 1. Baseline characteristics of study participants

\begin{tabular}{|c|c|c|}
\hline Baseline characteristics & GB & CON \\
\hline Age (yrs) & $42.9 \pm 3.4$ & $41.1 \pm 2.6$ \\
\hline BMI (kg/m2) & $31.9 \pm 1.8$ & $31.0 \pm 1.2$ \\
\hline Lean mass (kg) & $54.4 \pm 3.4$ & $52.9 \pm 2.8$ \\
\hline Fat mass $(\mathrm{kg})$ & $34.6 \pm 4.2$ & $34.0 \pm 3.1$ \\
\hline Waist circumference $(\mathrm{cm})$ & $102 \pm 4$ & $102 \pm 4$ \\
\hline $\operatorname{Sex}(M / F)$ & $2 / 10$ & $1 / 6$ \\
\hline $\mathrm{HbA} 1 \mathrm{C}[\%(\mathrm{mmol} / \mathrm{mol})]$ & $5.2 \pm 0.1(32.8 \pm 1.5)$ & $5.0 \pm 0.2(31 \pm 2.0)$ \\
\hline
\end{tabular}

Data are presented as mean \pm SEM unless specified otherwise.
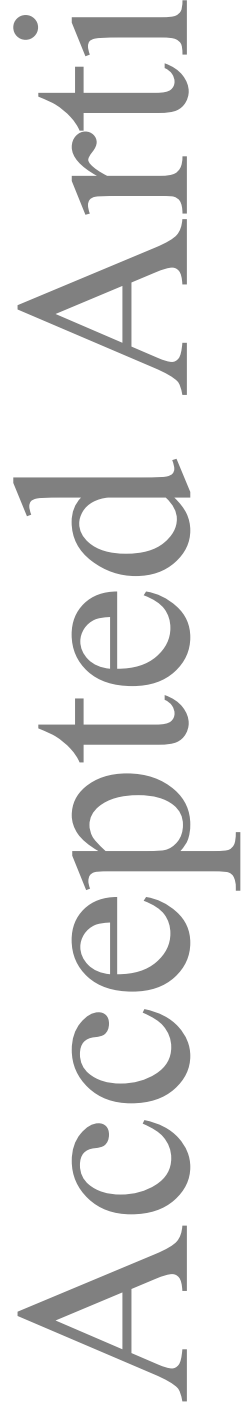
Table 2. Glucose and insulin response to increasing and decreasing intravenous glucose infusion in patients after gastric bypass (GB) and matched non-surgical controls $(\mathrm{CN})$

\begin{tabular}{|c|c|c|c|}
\hline & & GB & $\mathbf{C N}$ \\
\hline Glucose (mmol/l) & Fasting & $4.6 \pm 0.1$ & $4.8 \pm 0.2$ \\
\hline & Peak & $17.1 \pm 1.1$ & $13.7 \pm 0.9^{*}$ \\
\hline & AUC (40,160min) & $622 \pm 45$ & $542 \pm 43$ \\
\hline & AUC $(40,280 \mathrm{~min})$ & $1232 \pm 103$ & $718 \pm 69^{* *}$ \\
\hline Insulin (pmol/l) & Fasting & $24.2 \pm 6.7$ & $71.4 \pm 22.9^{*}$ \\
\hline & Peak & $470.9 \pm 140.9$ & $1368.7 \pm 320.6^{* *}$ \\
\hline$\bullet$ & AUC (40,160min) & $12595 \pm 3218$ & $41428 \pm 9182^{* *}$ \\
\hline & AUC $(40,280 \mathrm{~min})$ & $38269 \pm 9592$ & $94120 \pm 23710^{*}$ \\
\hline ISR (pmol/min) & Fasting & $73.1 \pm 11.6$ & $119.5 \pm 7.9^{*}$ \\
\hline & Peak & $581.1 \pm 93.7$ & $861.3 \pm 56.8^{*}$ \\
\hline & AUC (40,160min) & $22339 \pm 3923$ & $41914 \pm 2734^{* *}$ \\
\hline & AUC $(40,280 \mathrm{~min})$ & $51941 \pm 7562$ & $63536 \pm 7381$ \\
\hline$\beta$-cell glucose sensitivity & Step-up $(0,160 \mathrm{~min})$ & $33.5 \pm 5.5$ & $81.6 \pm 8.5^{\star *}$ \\
\hline$\left(\mathrm{pmol} \cdot \mathrm{min}^{-1} \cdot \mathrm{mM}^{-1} \cdot \mathrm{L}\right)$ & Step-down $(160,280 \mathrm{~min})$ & $31.0 \pm 5.6$ & $73.8 \pm 8.8^{* *}$ \\
\hline Insulin sensitivity $\left(10^{-4} \mathrm{dL}\right.$ & $\left.\mathrm{g}^{-1} \cdot \mathrm{min}^{-1} \cdot \mu \mathrm{U}^{-1} \cdot \mathrm{ml}\right)$ & $4.6 \pm 1.2$ & $3.1 \pm 1.1$ \\
\hline Glucose effectiveness (m & & $0.04 \pm 0.00$ & $0.07 \pm 0.01^{* *}$ \\
\hline
\end{tabular}

Data are presented as mean \pm SEM. ${ }^{*} p<0.05$ and ${ }^{* *} p<0.001$ compared with GB. 

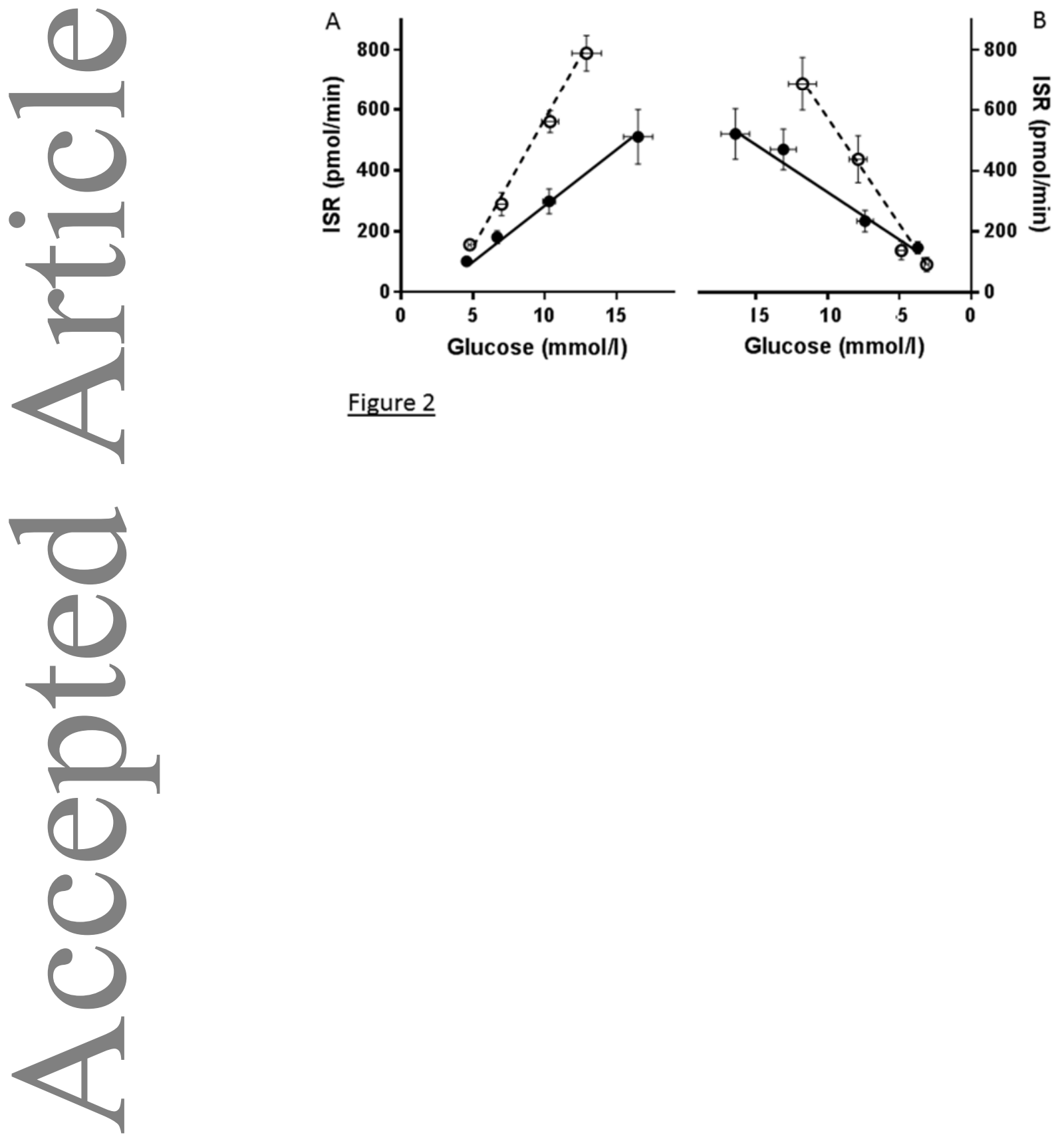

Figure 2 
